\title{
Shallow upper mantle rheology and secular ice sheet fluctuations
}

\author{
G. Spada ${ }^{\text {a,* }}$, F. Colleoni ${ }^{\text {b }}$, G. Ruggieri ${ }^{\text {a }}$ \\ a Istituto di Fisica, Università di Urbino "Carlo Bo", Urbino, Italy \\ b Centro Euro-Mediterraneo per i Cambiamenti Climatici, Bologna, Italy
}

\section{A R T I C L E I N F O}

\section{Article history:}

Received 1 July 2009

Received in revised form 2 December 2009

Accepted 29 December 2009

Available online 11 January 2010

\section{Keywords:}

Glacial isostasy

Glaciers and ice sheets

Generalized Maxwell rheology

\begin{abstract}
A B S T R A C T
On time scales from decades to centuries, continental cryospheric forcing in response to climate change constitutes a major source of isostatic disequilibrium that may influence future regional sea level variations. Current vertical displacements and gravity field variations are often estimated neglecting rheological effects and thus assuming a fully elastic response of the Earth. In this study, we adopt a more general point of view, aiming at describing ongoing surface movements resulting from recent glacial instabilities, also taking into account the effects associated with shallow upper mantle and crustal rheologies. Our computations are based on the Post-Widder Laplace inversion formula, which permits the straightforward computation of loaddeformation coefficients for steady state and transient rheologies up to very high harmonic degrees. Using a surface load with a simple geometry and time history, we compare the classical elastic solutions to those obtained considering the rheological response of the shallow upper mantle. While at the center of the ice sheet rheology only magnifies the elastic response, the pattern and time history of vertical displacement at the ice sheet margins show a greater complexity, mainly due to the development of lateral forebulges whose shape and amplitude are particularly sensitive to the rheology of the shallow upper mantle. In this region, assuming an elastic rheology is generally appropriate on a century time scale, but significant deviations from a purely elastic response (both sign and amplitude) are observed at longer time scales or when a low viscosity zone with Maxwell rheology is taken into account.
\end{abstract}

(c) 2010 Elsevier B.V. All rights reserved.

\section{Introduction}

Over the last few years there has been a rising interest in the possible effects of low viscosity crustal and asthenospheric layers upon the response of the Earth to surface loading. This was first motivated by geophysical evidence indicating a significant rheological stratification of the lithosphere (Ranalli and Murphy, 1987), and by seismic data suggesting the existence of a low velocity zone except beneath cold cratonic areas (Schubert et al., 2001). Second, the improved spatial resolution of ongoing satellite gravity missions motivates further research in this direction. According to Schotman (2008), results from the ESA's ongoing GOCE mission (http://www. esa.int/SPECIALS/GOCE/index.html) can be used in principle to detect spatial signatures to scales down to $\sim 100 \mathrm{~km}$, characteristic of crustal low viscosity zones (Vermeersen, 2003; van der Wal et al., 2004). Nowadays, the potential effects of these rheological features are investigated using various observations related to Glacial Isostatic Adjustment (GIA), ranging from Late Holocene relative sea level data (Kendall et al., 2003) to present-day geodetic measurements (Di Donato et al., 2000; Barletta et al., 2006), in an attempt to constrain

\footnotetext{
* Corresponding author. Istituto di Fisica, Università di Urbino "Carlo Bo", Via Santa Chiara n. 27, 61029 Urbino (PU), Italy. Tel./fax: +39 0722303389 (99).

E-mail address: giorgio.spada@gmail.com (G. Spada).
}

their thickness and lateral extent. As recently summarized by Schotman et al. (2009), adopted viscosity values for the "soft" crustal layers, with a typical thickness of $\sim 10 \mathrm{~km}$, may range between $10^{17}$ and $10^{20} \mathrm{~Pa} \mathrm{~s}$, i.e. at least one order of magnitude smaller than the traditional GIA value of $\sim 10^{21}$ Pa s (Haskell, 1935; Tushingham and Peltier, 1991; Spada et al., 1992). For the asthenosphere, similarly low viscosity values are used, consistently with those proposed in the seminal work of Cathles (1975) and in Fjeldskaar (1994).

Within the framework of linear rheologies, the response of the crust and of the shallow upper mantle to surface loads on decade to century time scales is further complicated by possible transient rheological effects (for which the strain rate is not constant in a creep experiment, see e.g. Christensen, 1982), which can be modeled using Generalized Maxwell Bodies (GMB). A well known example of transient rheology is given by the Burgers body, in which a steady state Maxwell and a transient Kelvin-Voigt elements are connected in series (Christensen, 1982; Ranalli, 1995). The resolving power of GIA observations appears to be insufficient to constrain the values of this four-parameter rheological body as well as of other possible GMBs (Yuen et al., 1986; Peltier et al., 1986; Körnig and Müller, 1989; Rümpker and Wolf, 1996). However, on decade to century time scales, it is now clear that transient rheological effects contribute significantly to the post-seismic deformation both on a regional (Pollitz, 2003) and on a global scale (Melini et al., 2008), and therefore they 
may influence the response of the Earth to recent cryospheric forcing, as first suggested by Sabadini et al. (1988) considering harmonic degree two signatures. For the sake of parsimony, in our study we will consider only one GMB in addition to the Burgers model, namely the Caputo rheology for which Körnig and Müller (1989) have determined a set of best-fitting parameters constrained by relative sea level observations in Fennoscandia and North America. The Caputo rheology (Caputo, 1967; Koeller, 1984), which is characterized by a stress-strain relationship containing fractional time-derivatives (e.g., Mainardi, in press), remains transient on all time scales. On the other hand, the transient relaxation of the Burgers body is limited to the Kelvin-Voigt element which is disposed in parallel to the Maxwell element in its mechanical analogue (e.g., Mainardi, in press).

The purpose of this paper is two-fold. First, by analyzing the response of a significant number of linear rheological models for the shallow upper mantle and the crust, we aim to determine how surface displacements, at a given harmonic degree, are amplified with respect to the elastic response on time scales from decades to centuries. This is addressed computing loading Love numbers (Love, 1909; Munk and MacDonald, 1960; Longman, 1962a,b; Farrell, 1972) for vertical and horizontal displacements by means of a recently re-discovered numerical method (Spada and Boschi, 2006; Spada, 2008) particularly suitable for complex viscosity profiles in the presence of transient rheological effects. Second, we study the effect of steady state and transient rheologies in the spatial domain, computing the vertical displacement field in response to the accretion of an ice sheet with simple geometry. In particular, we focus on the spatial pattern and the time scale of vertical displacements at the ice sheet margins, which are relevant to interpret present and future sea level changes in response to the current continental cryosphere fluctuations. Our results may have contribute to a better assessment of the effects of sudden ice melting upon future sea level rise, which is traditionally estimated using elastic (Mitrovica et al., 2009) or traditional viscosity models (Bamber et al., 2009).

\section{Methods}

All the computations have been performed using ALMA (Spada, 2008), a Love numbers calculator based on the Post-Widder (PW) inverse Laplace formula (Post, 1930; Widder, 1934; Widder, 1946). ALMA provides the response functions by-passing the computation of the viscoelastic "normal modes" (Spada and Boschi, 2006), which would demand the solution of a high-degree algebraic secular equation whose roots may be difficult to resolve by standard numerical methods (Vermeersen and Sabadini, 1997; Spada, 2008). The basic output of ALMA is constituted by the Heaviside Love numbers $h$ and $l$ (related to vertical and horizontal displacements, respectively) at a given harmonic degree, later employed in Section 1 to study the response of the Earth to finite-sized surface loads. In view of the geophysical applications in Section 2, the code has been subsequently modified in order to describe a more complex loading function.

Denoting by $\tilde{\mathrm{x}}(s)$ any of the Laplace-transformed Love numbers, the PW formula gives

$x(t)=\lim _{k \rightarrow \infty} \frac{(-1)^{k}}{k !}\left(\frac{k}{t}\right)^{k+1} \tilde{x}^{(k)}\left(\frac{k}{t}\right)$

(Post, 1930; Widder, 1934; Widder, 1946) where $t$ is time, $x(t)$ is the Love number in the time domain, superscript $(k)$ indicates the derivative of order $k$ and the dependence upon the harmonic degree is implicit to simplify notation. The discretization of the derivative and the convergence accelerators, needed to practically employ Eq. (1), are detailed in Spada (2008). Following previous experiments by Spada and Boschi (2006) and Spada (2008), carried out using multi- layered PREM models (Dziewonski and Anderson, 1981), all our computations have been performed in a multi-precision environment (Smith, 1989) using a number of significant digits $D=64$, and Gaver accelerators of order $N=8$ (Gaver, 1966; Valko and Abate, 2004). These settings are suitable also in the case of particularly challenging problems, such as the computation of very high-degree Love numbers for finely layered crustal models (see Section 3).

All the Earth models employed in this study, whose rigidity and density structures are shown in Table 1, share an incompressible rheology. The existence of gravitationally unstable Rayleigh-Taylor modes in compressible Earth models (see e.g., Hanyk et al., 1999) prohibits the use of the PW inversion method implemented in ALMA, which requires regularity of $\tilde{\mathrm{X}}^{(k)}$ along the real positive axis of the Argand plane (see Eq. (1)). Since this work mainly focuses on the response of the shallow upper mantle to short-term surface loading, we keep constant the lithosphere and lower mantle rheological parameters to values listed in Table 2 (we have directly verified that the thickness of the lithosphere has negligible effects upon the Love numbers compared to those produced by the rheological layering of the mantle).

The Earth models considered here can be classified into two distinct groups. The first, which encompasses Maxwell (i.e., steady state) rheological models, includes the elastic model, REF, LVZ and BRL. In the shallow upper mantle, REF and LVZ are characterized by a reference viscosity value of $10^{21} \mathrm{~Pa} s$ and by a low viscosity zone with viscosity ranging between $10^{18}$ and $10^{20} \mathrm{~Pa} \mathrm{~s}$, respectively. BRL is characterized by a finely layered crust that exactly reproduces the rheological structure described by Barletta et al. (2006). The second group accounts for upper mantle rheologies derived from two GMBs (Christensen, 1982; Körnig and Müller, 1989), namely Burgers (BUR, Burgers, 1935) and Caputo (CAP) bodies (Caputo, 1967; Wieczerkowski and Wolf, 1998). Differently from the first group, they are characterized by a transient behavior. However, while the response of CAP is fully transient, BUR combines steady state and transient behaviors (e.g., Mainardi, in press).

\section{Results}

The effects of shallow upper mantle rheological layering are first described in Section 1 by analyzing the response to individual harmonic components of loading. This is done considering the vertical $(h)$ and horizontal $(l)$ Love numbers in response to a Heaviside surface load history. Then, in Section 2, we simulate the vertical displacements induced by the slow accretion of an ice sheet of parabolic crosssection, in order to determine the time scales of relaxation when a full spectrum of harmonics are simultaneously accounted for. We focus both on vertical movements at the center and at the margins of the ice sheet. In the context of global climate change, these latter movements are relevant to regional relative sea level variations in response to present and future glaciers and ice sheet fluctuations (Sheperd and Wingham, 2007).

Table 1

Density and rigidity profiles for the elastic model and for REF, LVZ, BUR and CAP (see first column of Table 2). The numerical values are obtained by volume-averaging the PREM model of Dziewonski and Anderson (1981). Rheological features of mantle layers are described in Table 2.

\begin{tabular}{llcl}
\hline Layer & $\begin{array}{l}\text { Radius range } \\
(\mathrm{km})\end{array}$ & $\begin{array}{l}\text { Density, } \rho_{0} \\
\left(\mathrm{~kg} \mathrm{~m}^{-3}\right)\end{array}$ & $\begin{array}{l}\text { Rigidity, } \mu_{0} \\
\left(10^{11} \mathrm{~Pa}\right)\end{array}$ \\
\hline Lithosphere & $6281-6371$ & 3192 & 0.596 \\
Shallow upper mantle & $5971-6281$ & 3427 & 0.720 \\
Transition zone & $5701-5971$ & 3857 & 1.064 \\
Lower mantle & $3480-5701$ & 4877 & 2.194 \\
Core & $0-3480$ & 10931 & 0.00 \\
\hline
\end{tabular}


Table 2

Shallow upper mantle complex rigidity and rheological parameters used in this study (radius in the range $5971 \leq r \leq 6281 \mathrm{~km}$ ). Except for BRL, all these models include a homogeneous $90-\mathrm{km}$ thick elastic lithosphere, a lower mantle with viscosity $2 \times 10^{21} \mathrm{~Pa} \cdot \mathrm{s}$, and a homogeneous inviscid core (see also Table 1 ).

\begin{tabular}{|c|c|c|c|}
\hline Model & Rheology & Rigidity, $\tilde{\mu}(s)$ & Material parameters \\
\hline Elastic $^{\mathrm{a}}$ & Elastic & $\mu$ & $\mu=\mu_{0}$ \\
\hline REF & Maxwell & $\frac{\mu s}{s+\mu / \eta}$ & $\mu=\mu_{0}, \eta=10^{21} \mathrm{~Pa} \mathrm{~s}$ \\
\hline LVZ & $" ”$ & & $\mu=\mu_{0}, \eta=10^{18} \div 10^{20}$ Pa s \\
\hline $\mathrm{BRL}^{\mathrm{b}}$ & $" ”$ & $\frac{\mu(r) s}{s+\mu(r) / \eta(r)}$ & See Barletta et al. (2006) \\
\hline BUR & Burgers & $\frac{s^{2}+s\left(\frac{\mu}{\eta}+\frac{\mu+\mu^{\prime}}{\eta^{\prime}}\right)+\frac{\mu \mu^{\prime}}{\eta^{\prime}}}{\eta^{\prime}}$ & $\begin{array}{l}\mu=\mu_{0}, \eta=10^{21} \text { Pa s } \\
\mu^{\prime}=0.3 \mu_{0}, \eta^{\prime}=0.1 \eta\end{array}$ \\
\hline $\mathrm{CAP}^{\mathrm{b}}$ & Caputo & $\mu_{0} \frac{\left(s T_{c}\right)^{\gamma}}{1+\left(s T_{c}\right)^{\gamma}}$ & $\tau_{c}=6.3 \cdot 10^{9} \mathrm{~s}, \gamma=0.8$ \\
\hline
\end{tabular}

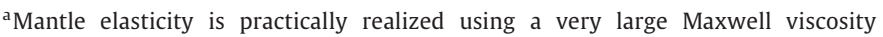
$\left(\eta=10^{50}\right.$ Pa s).

${ }^{\mathrm{b}}$ The BRL model is characterized by complex, piecewise radially dependent rigidity $\mu=\mu(r)$ and viscosity $\eta=\eta(r)$ in the crust and in the shallow upper mantle, as described in Table 2 of Barletta et al. (2006).

${ }^{\mathrm{c}}$ The numerical value of the time scale $\tau_{c}$, adopted from Körnig and Müller (1989), is close to the $\eta / \mu$ ratio (i.e., the intrinsic Maxwell relaxation time for the upper mantle) for the REF model.

\subsection{Spectral domain}

In this section we consider separately the effects of three important rheological features: i) a low viscosity zone in the shallow upper mantle, ii) transient components of mantle relaxation, and iii) ductile crustal layers. The time evolution of vertical $(h)$ and horizontal ( $l$ ) Love numbers for the fundamental mode of harmonic degree $n=2$ which correspond to these three rheological models is shown in the left frames of Figs. 1-3, respectively. Because we have verified that the sensitivity of the gravitational potential Love number $(k)$ to rheological stratification in the upper mantle is comparable to that of $h$, we will only display numerical results concerning $h$ and $l$. Since Eq. (1) by-passes the computation of the viscoelastic normal modes (Spada and Boschi, 2006), the analysis in the spectral domain cannot be performed looking at individual relaxation modes and time scales, as done by Rümpker and Wolf (1996) and previously, for a spherical Earth, by Yuen et al. (1986) and Peltier et al. (1986). Since the Post-Widder formula does not provide the relaxation time scales of the Earth model (Spada and Boschi, 2006), we estimate them studying the Heaviside Love numbers, which provide the "creep response" (Christensen, 1982) of our Earth model. Of course, estimates of the relaxation times could be performed by least squares methods, as done by Hanyk (1999).

To quantify the effects of rheological layering upon a wide range of harmonic degrees, in the right frames of Figs. 1-3 we show the rheological amplification ratio (hereafter RA), defined as

$R A_{n}(t)=\left|\frac{x}{x^{e}}\right|$

where $n$ is harmonic degree, $x$ indicates any of the Heaviside Love numbers ( $h$ or $l$ ) for a given viscoelastic rheology while $x^{e}$ is the same Love number computed for a purely elastic Earth (i.e., neglecting the effects of viscoelastic relaxation).

\subsubsection{Low viscosity zone}

Fig. 1a and c shows the time evolution of the vertical and horizontal Love numbers at harmonic degree $n=2$ for the REF and two LVZ models (characterized by a low viscosity shallow upper mantle), respectively. The Love numbers, displayed in a very broad time window, are characterized by short and long time scales asymptotes corresponding to their "elastic" $(t \mapsto 0)$ and "fluid" $(t \mapsto \infty)$ responses, and intermediate fluctuations whose amplitude and time scale reflect the rheological layering of the Earth model (see e.g., Vermeersen and Sabadini, 1997). Elastic response only depends on the elastic structure, given in Table 1, which does not change between the models; fluid response only depends on buoyancy effects due to internal
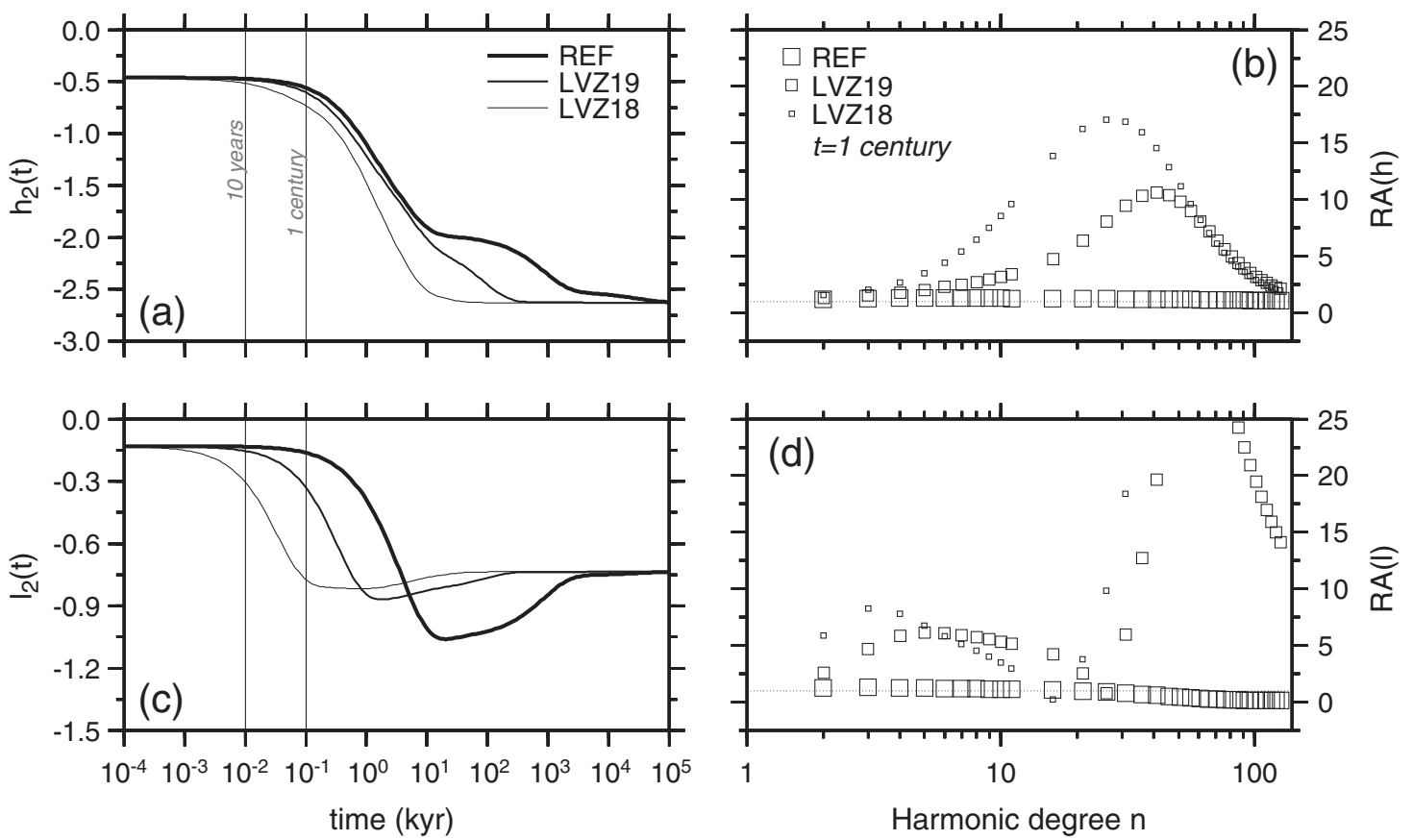

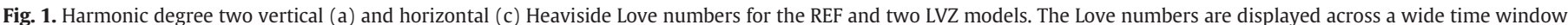

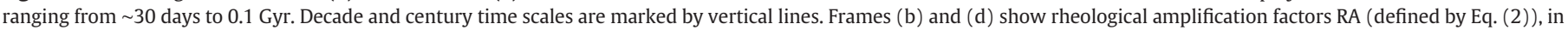

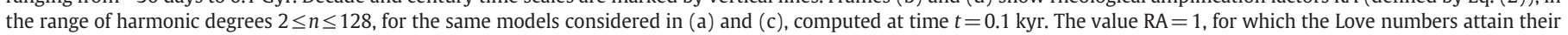
elastic amplitudes, is marked by a dotted horizontal line. 

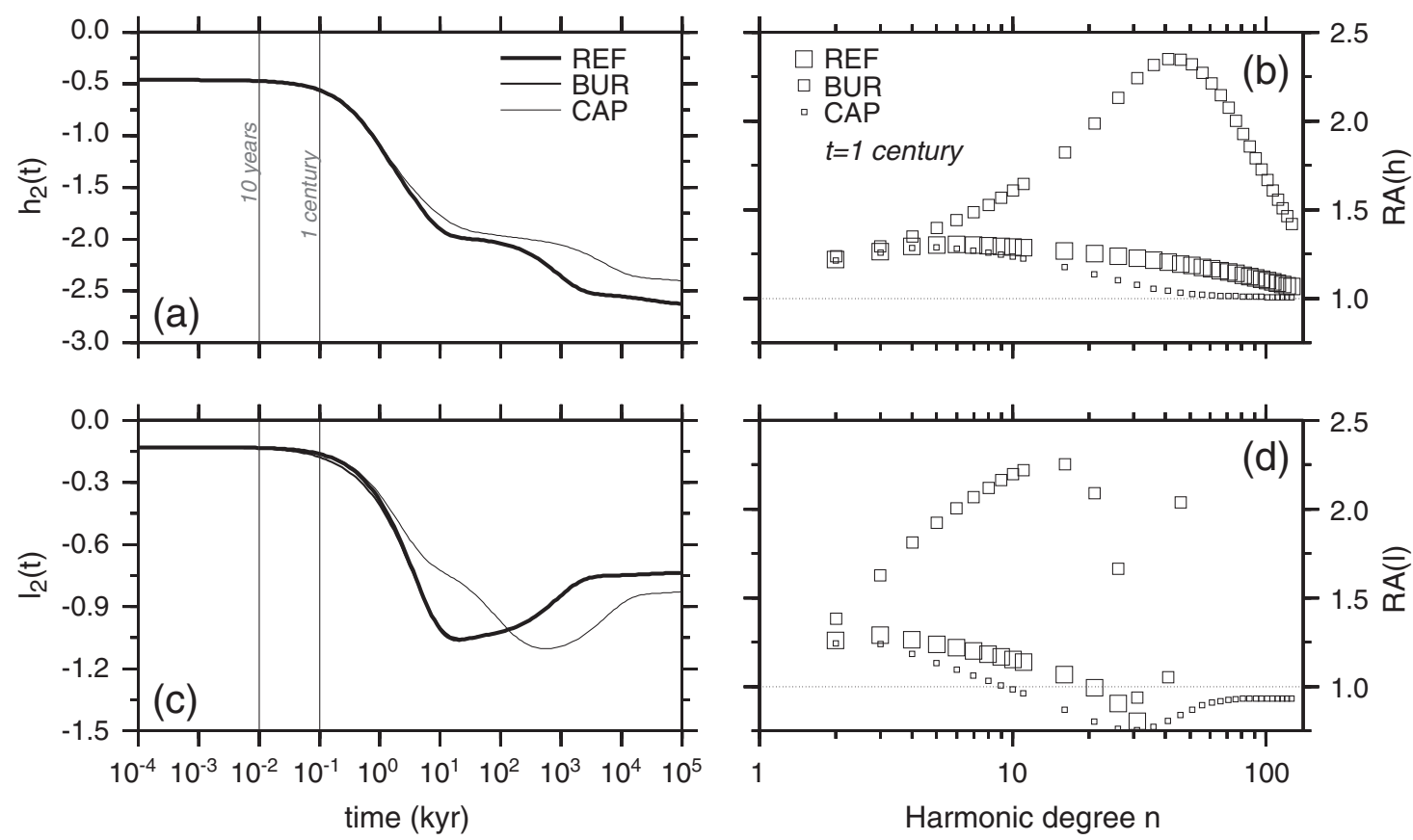

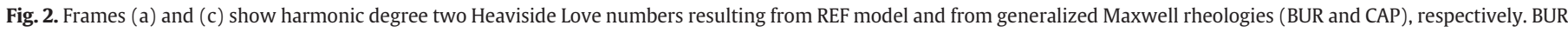

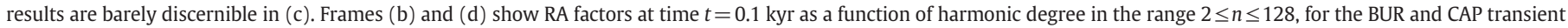
rheological models.

density contrasts and on the flexural rigidity of the elastic lithosphere. Hence, for a given Earth structure (i.e., density and rigidity profile) elastic and fluid responses are insensitive to prescribed viscosity values and therefore the asymptotes in Fig. $1 \mathrm{a}$ and $\mathrm{c}$ are the same for the LVZ and REF models.

Since our interest focuses on short-term ice sheet fluctuations, in Fig. 1a we have indicated with vertical lines on the decade and on century time scales. For the REF model, characterized by a mantle viscosity profile that has been long employed in the GIA community (i.e., $10^{21} \mathrm{~Pa} \mathrm{~s}$ in the shallow upper mantle and transition zone, and $2 \times 10^{21} \mathrm{~Pa} s$ in the transition zone and lower mantle, respectively, see Tushingham and Peltier, 1991) these long-wavelength Love numbers do not show significant deviations from their elastic asymptotes, even on a century time scale. However, as expected from basic physics, the opposite is true when a LVZ is accounted for, due to the shorter Maxwell relaxation times involved. In this case, the degree two responses deviate from the elastic values, even on the time scale of a few decades. Horizontal movements (Fig. 1c) show a larger sensitivity
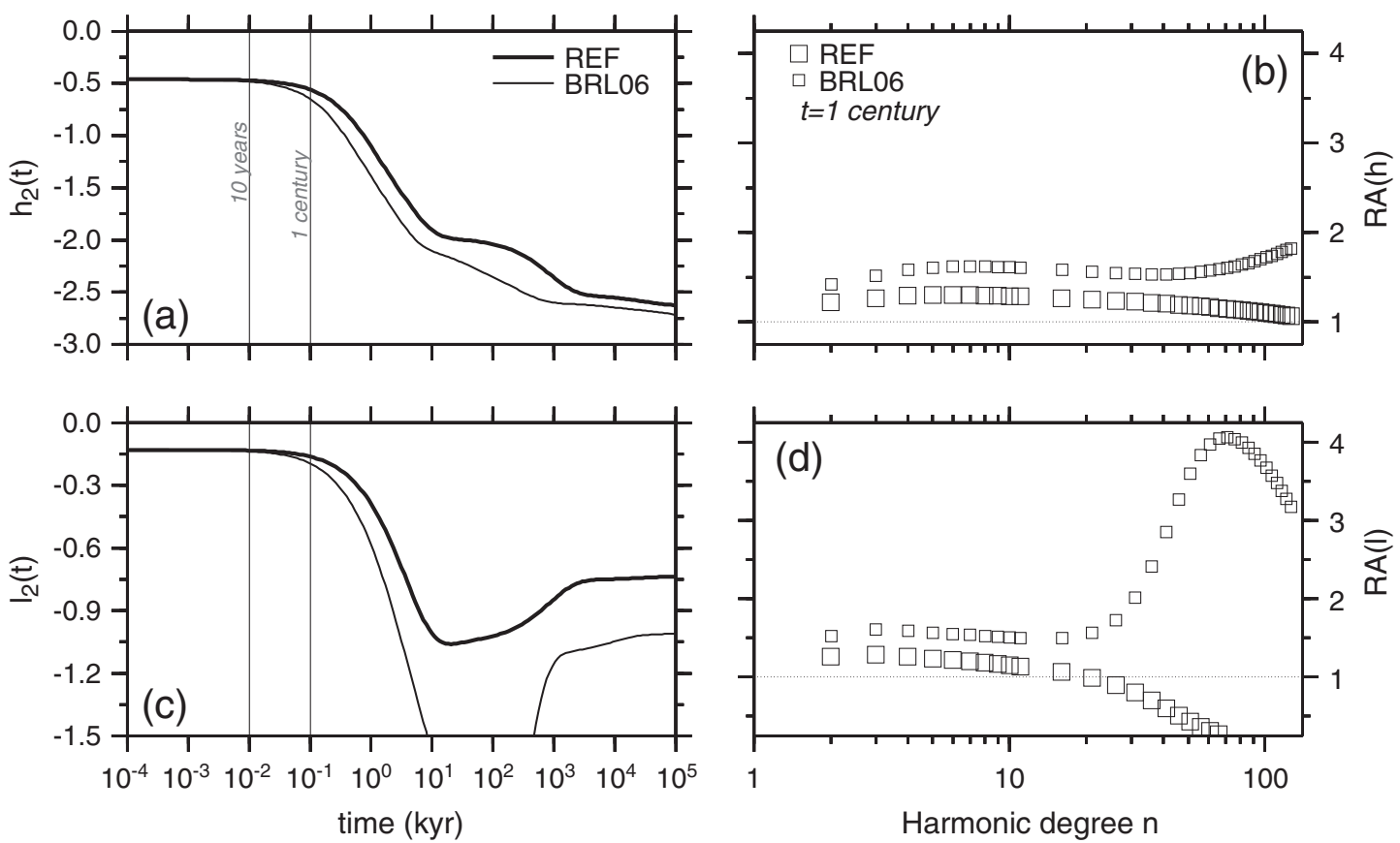

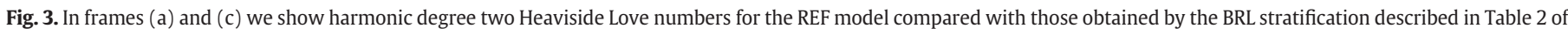

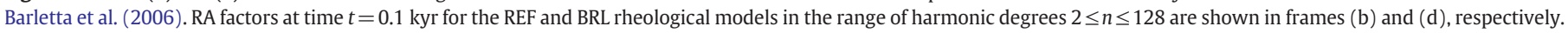


to the presence of the LVZ compared to vertical ones (1a), in agreement with previous GIA investigations (e.g., Vermeersen and Sabadini, 1997).

Fig. $1 \mathrm{~b}$ and $\mathrm{d}$ show the ratio RA at time $t=1$ century for models REF and LVZ. Mantle rheology generally imposes an amplification of the elastic Love numbers ( $R A \geq 1$ ) on the range of harmonics considered here $(2 \leq n \leq 128)$. For LVZ, peak values $(R A \geq 10)$ are obtained for $20 \leq n \leq 50$ for the vertical Love number (Fig. 1b) and even larger effects for $n \geq 20$ are observed on the horizontal ones (Fig. 1d). Some features of the RA curves have a straightforward explanation. For instance, the relatively small value of $R A(h)$ for low $n$ values, (Fig. 1b), is justified by the larger sensitivity of low degree harmonics of vertical displacements to the bulk viscosity of the mantle (Vermeersen and Sabadini, 1997) rather than to relatively small, shallow features as the LVZ. Due to flow channeling, horizontal motions are more affected by the presence of a LVZ, especially for low $n$ values, consistently with Fig. 1a and c. This agrees with previous results (see e.g., Spada et al., 1992) since the importance of horizontal displacements was first recognized by James and Morgan (1990). The trend of all RA curves is to converge for large $n$ values, when the sublithospheric rheology tends to be less important, and the REF and LVZ models are expected to provide comparable (and essentially elastic) responses. Due to the relatively thin lithosphere, however, convergence indeed occurs for $n \gg 128$, the maximum degree considered. The largest values of $R A(h)$ are obtained for LVZ18. This is not the case for $l$ (Fig. $1 \mathrm{~d}$ ), for which the sensitivity to the LVZ viscosity varies considerably with $n$. For $n \approx 20, R A(l)$ values for LVZ approach REF, and $l$ changes its sign (this causes the cusp feature in Fig. 1d).

\subsubsection{Generalized Maxwell rheologies}

The response of the Earth to a harmonic degree two surface load in the presence of transient rheological effects is considered in Fig. 2. Numerical values of the material parameters for the Burgers (BUR) and Caputo (CAP, showing a transient behavior at all times) GMBs are given in Table 2. Instead of exploring the parameters space of these two rheologies, as done by (Rümpker and Wolf, 1996) in a flat Earth approximation, here we use the best-fitting values determined in the case of Fennoscandia from the study of Körnig and Müller (1989) (notice, however, that the transient viscosity for the Burgers body is reduced by a factor of ten with respect to the preferred value of Kornig and Müller, which facilitates the study of short-times effects). We limit our attention to the BUR and CAP GMBs, leaving the study of the response of the Lomnitz and of the $Q$ power-law body (see e.g., Körnig and Müller, 1989) to future investigations.

Comparison between Fig. $2 \mathrm{a}$ and $\mathrm{c}$ with the corresponding frames of Fig. 1 shows that long-wavelength vertical displacements are by far more sensitive to the Maxwell LVZ than to BUR or CAP transient rheologies. No deviations from the reference model are basically observed even on millennia time scales, and these effects are found to be small for longer periods. BUR results are superposed to REF on all time scales. These findings appear at odds with the analysis of Sabadini et al. (1988), who pointed the importance of mantle relaxation to low degree harmonic loads (up to $n=9$ ) even for time scales shorter than a century. However, the results of Sabadini and colleagues pertain to the lower mantle, while here we are only concerned with transient components of shallow upper mantle rheology, which are expected to produce significant effects upon deformations of shorter wavelength. Furthermore, it is well possible that rheological transient parameters different from those employed here may provide a larger amplification of the harmonic degree two response.

The time evolution of the harmonic degree two horizontal Love number, displayed in Fig. 2c, shows some interesting features. In the time window between one decade and one century, no significant relaxation is observed for the CAP solution, which matches closely the REF results. The BUR results are virtually indistinguishable from REF at all time scales. A remarkable finding is the very long time scale required for the CAP model to reach isostatic equilibrium at this harmonic degree, certainly exceeding $10^{5}$ kyrs. However, a isostatic limit will certainly be reached even if CAP is transient at all times, since the CAP complex shear modulus $\tilde{\mu}(s)$ vanishes for long times (i.e., when the complex variable $s \mapsto 0$ ) as in the case of the steady state Maxwell rheology (see Table 2). A similar, but less pronounced, departure from equilibrium is also observed for vertical movements (see Fig. 2a). The physical reason for the long-standing global isostatic disequilibrium imposed by the CAP rheology is unclear at this stage. From further computations, not shown here, we find that such a long relaxation is not motivated by the form of the effective shear modulus and the numerical values of upper mantle rheological parameters adopted here (see Table 2). We therefore argue (but a rigorous demonstration would demand the computation of internal stress fluctuations) that the Caputo rheology enhances the degree of mechanical shear coupling between lower and upper mantle, also possibly delaying the return to equilibrium of compositional internal boundaries which are known to be intrinsically characterized by long relaxation times (e.g., Spada et al., 1992).

The RA factors for generalized Maxwell rheologies, shown in Fig. 2b and d, clearly indicate that model CAP is characterized by a modest amplification at all wavelengths with RA close to 1 , which makes it similar to REF on the century time scale. For high-degree vertical displacements, CAP is more elastic than REF, while for horizontal displacements, the CAP results are always found below the elastic response. A more complex response is exhibited by BUR, with a response similar to LVZ19 for vertical movements (compare with Fig. 1 b), but an amplitude reduction by a factor of $\sim 4$. Similarly to the LVZ results, BUR RA factors for horizontal love numbers are bimodal as a function of degree, but their amplitude is greatly reduced.

\subsubsection{Ductile crustal layers}

Amongst the various models characterized by a ductile crust available from the literature (see the Introduction and the review of the "CLVZ" models in Schotman, 2008), here we consider the one proposed by Barletta et al. (2006) in their study about the vertical uplift in response to the shrinkage of the current alpine glaciers. This model (here referred to as BRL) is inspired to the work of Burov et al. (1999), based on thermo-mechanical modeling of crustal and lithospheric structures beneath the alpine chain. BRL is characterized by a $18.5-\mathrm{km}$ thick effectively elastic upper crust (Maxwell viscosity is $10^{35} \mathrm{~Pa}$ ), a $11.5-\mathrm{km}$ ductile crust with viscosity of $2.15 \times 10^{19} \mathrm{~Pa} \mathrm{~s}$, a further $10-\mathrm{km}$ thick crustal layer that includes the Moho, with viscosity of $5 \times 10^{21} \mathrm{~Pa}$ s, and a wide, 630-km thick, low viscosity upper mantle with viscosity $4.64 \times 10^{20} \mathrm{~Pa}$ s. Deeper down, mantle viscosity takes the classical "Haskell value" $\left(10^{21} \mathrm{~Pa}\right.$ s). Hence, differently from our REF model (see Table 1), BRL does not include an elastic lithospheric mantle, and its sub-crustal low viscosity region is $\sim 2$ times thicker than the REF shallow upper mantle. Numerical values of rheological and physical parameters of BRL, essentially inspired to Burov et al. (1999), are given in Table 2 of Barletta et al. (2006) and will not be repeated here. We are aware that more sophisticated approaches are however possible to describe the rheology of the crust, using the theory of composite materials to account for the presence of rheological heterogeneities (Ivins and Sammis, 1996), and consequently including transient rheological effects.

From Fig. 3a and $\mathrm{c}$ it is apparent that significant departures of the BRL results relative to REF are only possible on time scales of approximately one century, as far as we consider the harmonic degree two response. Both vertical and horizontal displacements show a remarkably long relaxation time (longer than the one observed using LVZ models in Fig. 1), with the latter greatly amplified with respect to REF due to the shear weakness of the relatively thick low viscosity sub-crustal layers in model BRL. With increasing time, deviations of 
BRL with respect to REF can be attributed to the delayed flow in the ductile crustal layer.

The RA factors for BRL model, shown in Fig. $3 \mathrm{~b}$ and $\mathrm{d}$ at time $t=1$ century, indicates that with the rheological parameters employed for this model, we should not expect dramatic viscoelastic amplifications of vertical movements (RA $\leq 4)$. This is fairly consistent with the results of Barletta et al. (2006). Nevertheless, horizontal movements, not considered in the analysis of Barletta et al. (2006), show a large peak in the RA factor for $n \approx 100$, which may have consequences on the interpretation of regional horizontal deformations pattern in the alpine region (Stocchi et al., 2005). The peak of $R A(l)$, already noticed by Klemann and Wolf (1999), can be attributed to the buoyancy mode associated with the density jump across the ductile crustal layer, which is also responsible for the observed increase of $R A(h)$ for large $n$.

For small scale surface loads, such as those involved in the mass variations occurring across the Alps, a high-resolution approach is required, especially for the assessment of vertical movements in the near field of the glaciers, as pointed by Barletta et al. (2006) (of course, the flat Earth approximation may constitute a valid alternative approach in this context, as discussed by Rümpker and Wolf, 1996). Our implementation of the traditional viscoelastic normal modes method may fail for large harmonic degrees (Spada, 2008) and failures are indeed possible even for fundamental, low degree harmonics (Spada and Boschi, 2006). For these reasons, in order to solve this challenging numerical problem, we use for the first time our numerical implementation of the PW formula for the computation of Love numbers of very large harmonic degree. Validation tests of the PW formula are given in Spada and Boschi, 2006. Vertical (left) and horizontal (right) Love numbers for the BRL model, displayed in Fig. 4, correspond to the elastic and fluid regimes, respectively, for degrees in the range $10 \leq n \leq 10^{5}$. As far as $n$ exceeds $\sim 2000$, fluid and elastic responses practically coincide, indicating that at these wavelengths the viscoelastic relaxation is totally prevented by the flexure of the elastic crustal layer. Differently from Barletta et al. (2006), very large degree Love numbers have not been extrapolated from values obtained for smaller $n$ values, but directly computed using software ALMA (Spada, 2008). For the vertical Love number, the asymptotic value $h=-3.82$ computed by Barletta et al. (2006) is matched to within $1 \%$. For the horizontal Love number, no comparison with independent computations is presently possible; from Fig. 4, we observe that product $n l$ vanishes for $n \mapsto \infty$.

\subsection{Spatial domain}

Once the Love numbers are computed, the displacements in the spatial domain can be retrieved by standard methods (see e.g. Spada, 2003). We will focus on vertical displacement, since this is directly related to regional sea level variations. Instead of solving the sea level equation in its complete form (Spada and Stocchi, 2006), we neglect the effects from oceans self-gravitation, which in the near field of the load are blurred by the direct effects of surface loading. This approximation has been also adopted and discussed in the numerical study of Spada et al. (2006). However, differently than in Spada et al. (2006), here vertical displacements account for self-gravitation of the solid Earth (i.e., the " $k$ " loading Love number is $k \neq 0$ ), whose effects are not negligible for long-wavelength deformation ( $\mathrm{Wu}$ and $\mathrm{Ni}, 1996$ ). Assuming axially symmetric surface load, sea level change is

$S(\theta, t)=-U(\theta, t)+S^{E}(t)$

where $U$ is vertical displacement, $S^{E}$ is the eustatic (i.e., spatially uniform) component of sea level change, and $\theta$ is colatitude with respect to the center of the load (see e.g., Spada and Stocchi, 2006). While $S^{E}(t)$ is only dependent upon variations of the mass of the ice sheets, vertical displacement is sensitive to rheology. The expression of the Laplace-transformed vertical displacement is

$\tilde{U}(\theta, s)=\frac{3}{\rho_{e}} \sum_{n=0}^{n_{\max }} \frac{\sigma_{n}}{2 n+1} \tilde{h}_{n}(s) \tilde{f}(s) P_{n}(\cos \theta)$

where $\rho_{e}$ is the average density of the Earth, $\sigma_{n}$ is the spectral density of the surface load and $P_{n}(\cos \theta)$ is Legendre polynomial of harmonic degree $n$ (Spada, 2003). Here we use a load with a parabolic profile of half-amplitude $\alpha$ (see Equation 3.78 in Spada, 2003). Since the load profile is continuous (but its gradient is not) for this geometry, the Gibbs phenomenon is mitigated. Hints on how to deal with load profiles including jump discontinuities are given by Barletta et al. (2006). Due to the factor $(2 n+1)$ at the denominator of Eq. (4) and to the $n$-dependence of $\sigma_{n}$ and $P_{n}(\cos \theta)$, the sensitivity of $\tilde{U}(\theta, s)$ to the shallow upper mantle rheology cannot be simply guessed from the spectral Love numbers analysis of Section 1.
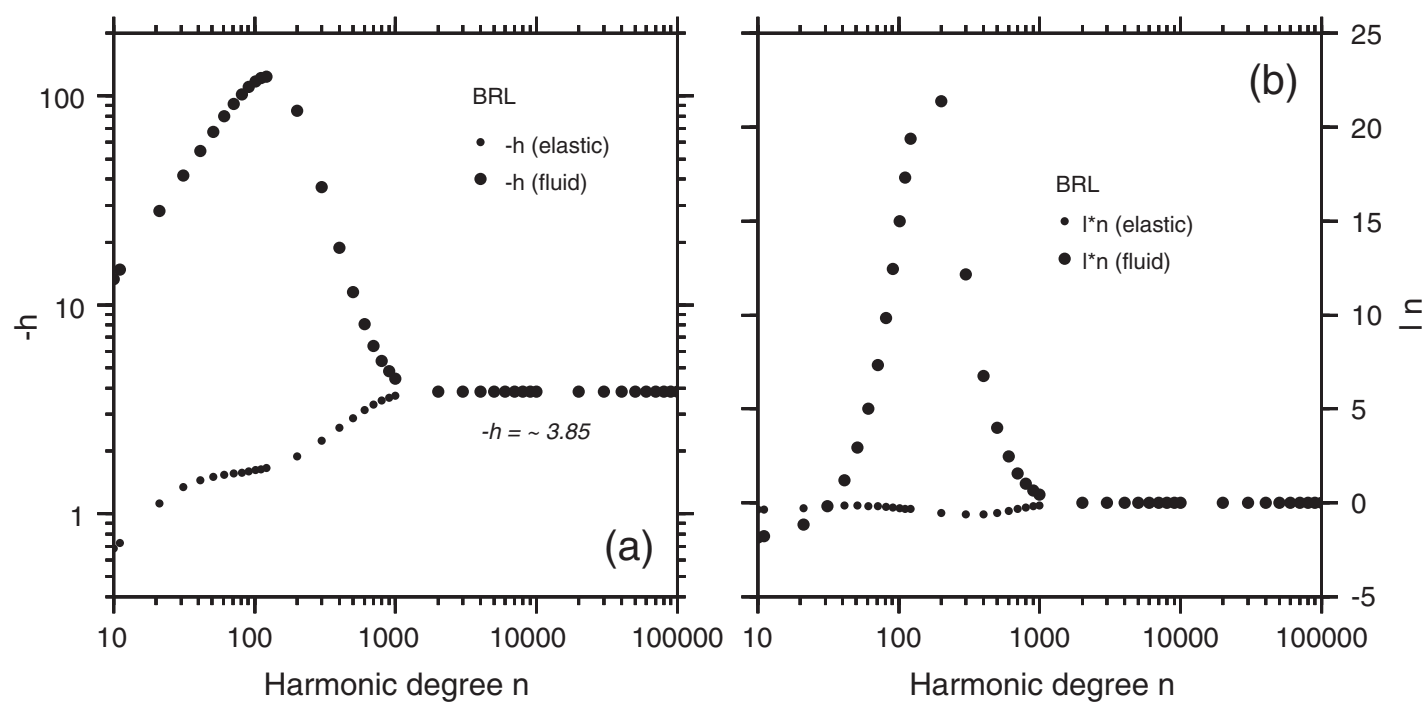

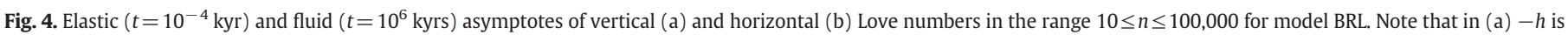
shown and the $y$-axis is logarithmic. In (b), the product $l \cdot n$ is shown. 
Function $\tilde{f}(\mathrm{~s})$ represents the Laplace transform of the time history of the surface load:

$\tilde{f}(s)=\frac{1}{s} \cdot \frac{1-\exp (-s \tau)}{s \tau}$,

where $\tau$ is the duration of the accretion phase (for $\tau \mapsto 0, \tilde{\mathrm{f}}(s) \mapsto 1 / s$, the Laplace transform of the Heaviside step function $H(t)$ ). The final maximum thickness of the ice sheet is chosen so that its accretion occurs at the constant rate of $10 \mathrm{~cm} \mathrm{yr}^{-1}$ during a time period of $\tau=1 \mathrm{kyr}$ (these rates are comparable with current elevation rates observed in some coastal areas of Antarctica, see Sheperd and Wingham, 2007, and references therein). Eq. (4) is inverted in the time domain using the PW formula given by Eq. (1). Of course, the meaning of the results below, and particularly the sensitivity of $U(\theta, t)$ to upper mantle rheology, would be unchanged in case of melting (in the case of melt, the rates of displacements would simply reverse sign).

The vertical displacement obtained using Eq. (4) is shown in Fig. 5 as a function of $\theta$, at time $t=0.3 \mathrm{kyr}$ after the beginning of loading, using $n_{\max }=512$. Here $\alpha=15^{\circ}$, which results in a disc load comparable to the Antarctic ice sheet (the effects of varying $\alpha$ will be considered at the end of this section). The results, shown in Fig. $5 \mathrm{a}$ and b for classical Maxwell and GMBs, respectively, indicate that significant departures from the elastic solution (dashed curves) are expected for many of the rheological models considered, at all the spatial scales. The REF solution is qualitatively similar to the elastic one that shows a slightly enhanced amplitude and negative values on the whole range of colatitudes. Introducing additional rheological features, enhances local compensation and amplifies the deformations beneath the load by a factor ranging between 1 and $\sim 3$. In addition, we observe a modification of the pattern of vertical movements at the ice sheet margins, showing, for LVZ18 and LVZ19, the development of
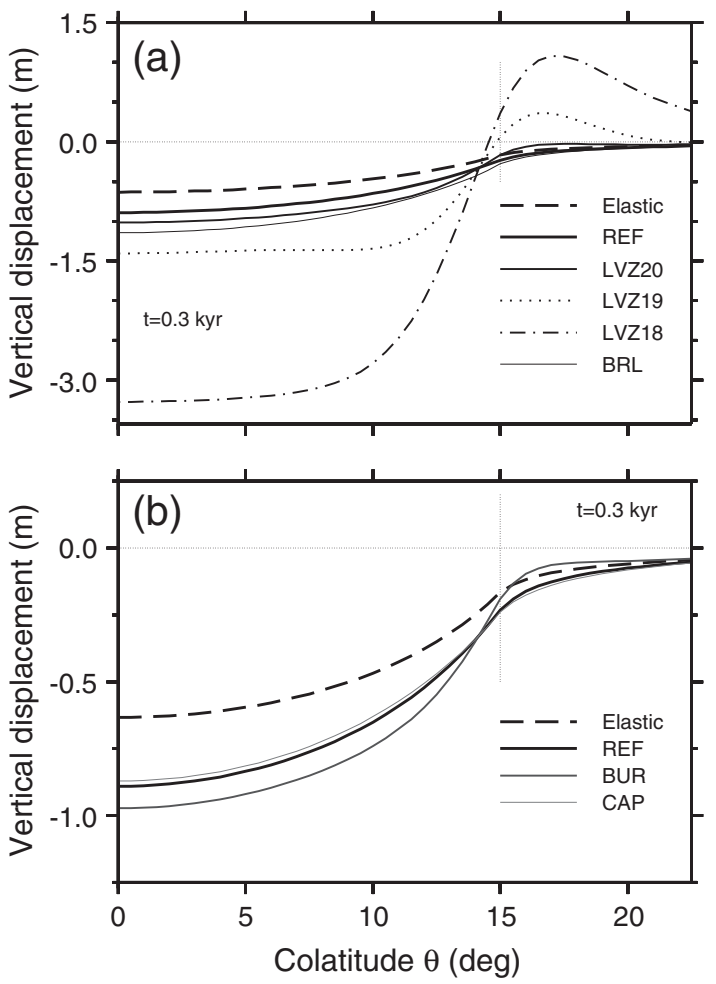

Fig. 5. Vertical displacement, computed by Eq. (4) at time $t=0.3 \mathrm{kyr}$ beneath the load and in its near field periphery, for the Maxwell (a) and generalized Maxwell rheologies (b) considered in this study, compared with elastic and REF solutions. The ice sheet extent $(\theta=\alpha)$ is marked by a vertical dashed line. lateral forebulges whose amplitude amounts to $\sim 1 / 3$ of the central subsidence; for a stiffer low viscosity zone (LVZ20), the response is similar to REF. On the time scales considered here, forebulges do not develop when generalized Maxwell rheologies are considered.

The history of uplift at the ice sheet center $(\theta=0)$ is shown in Fig. 6, where we consider separately Maxwell (a) and generalized Maxwell rheologies (b), in a time window of $0.5 \mathrm{kyr}$. On these time scales, the rate of deformation as the ice sheets develops is nearly constant for all the models considered, except for LVZ18, which clearly shows an acceleration. As expected on physical grounds, rates of deformation at the ice sheet center increase monotonously with decreasing bulk viscosity in the shallow upper mantle and it is apparent that deviations from the elastic predictions can only be significant after a few centuries from the onset of the glacial episode, unless we assume model LVZ18. Fig. $6 \mathrm{~b}$ shows that the sensitivity of central deformation to transient rheological components is modest, since BUR and CAP results are basically indistinguishable from the REF response. However, on the whole time scale considered, the total viscoelastic displacement the GMB rheologies amounts to $\sim 2$ times the elastic displacement.

The history of vertical deformation at the ice sheet margin $(\theta=\alpha)$ is shown in Fig. 7, for Maxwell (a) and generalized Maxwell rheologies (b), respectively. The elastic solution (thick dashed line), which follows the linear load time history, indicates vertical velocities close to $0.5 \mathrm{~mm} \mathrm{yr}^{-1}$. The introduction of a rheological layering in the shallow upper mantle may alter the elastic response in two ways. First, the latter can be subjected to a viscoelastic amplification, without showing a significant acceleration in the time period considered. Such apparently elastic response, also observed by deformations at the load center (Fig. 6), can be noted when the Maxwell REF and BRL models are employed (Fig. 7a), but also when transient rheologies are considered (BUR and CAP, Fig. 7). Remarkably, for all generalized Maxwell rheologies the responses stand below the elastic solution, which indicates that these GMBs always imply, at the load margins, an amplification of
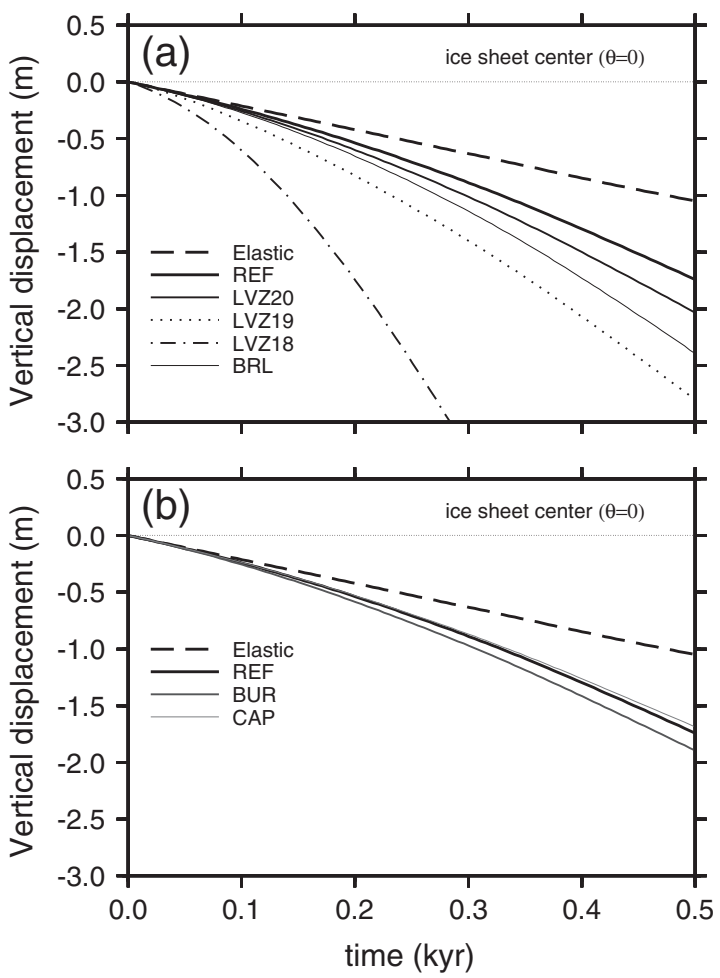

Fig. 6. Vertical movements at the center $(\theta=0)$ of the ice sheet considered in Fig. 5, as a function of time after the beginning of loading, for Maxwell (a) and generalize Maxwell rheologies (b). 

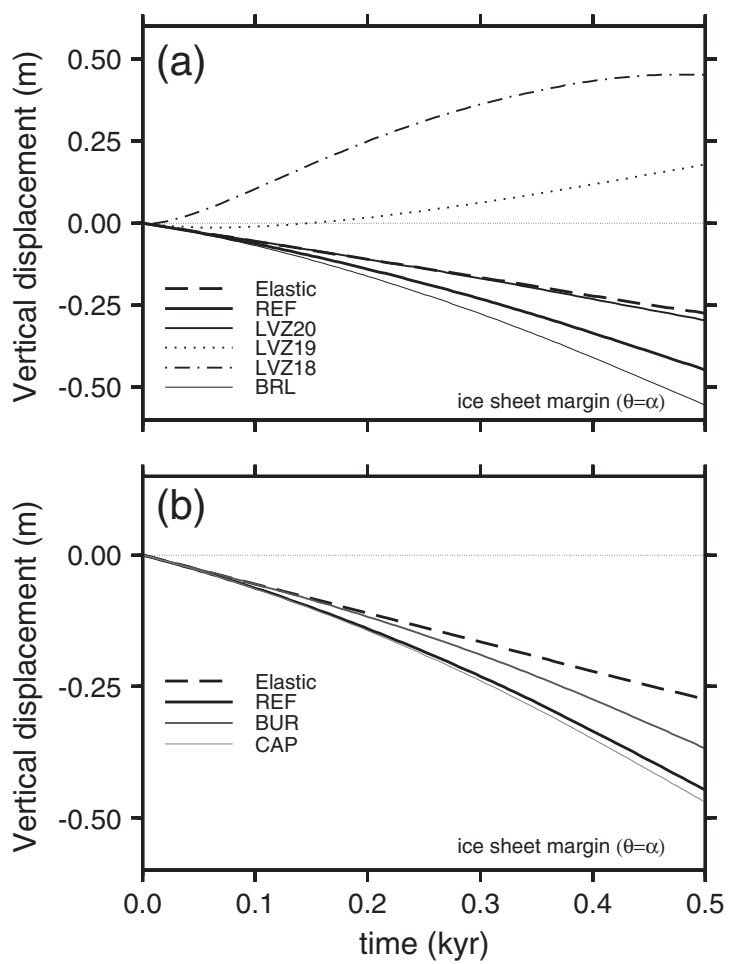

Fig. 7. Vertical movements at the margin $(\theta=\alpha)$ of the ice sheet considered in Fig. 5, as a function of time after the beginning of loading. Frames (a) and (b) show results for Maxwell and generalized Maxwell rheologies, respectively.

the elastic response of the Earth (this is indeed also true at the center of the load, see Fig. 6). The second modification of the elastic response is observed in Fig. 7a for the LVZ models. On one hand, for LVZ19, our predictions show vanishing vertical movements during the first century, and a subsequent deformation at a rate comparable to REF, but with opposite sign (uplift). LVZ18, on the other hand, produces an uplift since the onset of loading, characterized by a complex time evolution. For LVZ20, the history of vertical displacement closely follows the REF predictions.

From GIA investigations (e.g., Spada et al., 1992), we know that the response to surface loading is expected to depend, beside mantle rheological layering, upon the size of the load compared to the thickness of the elastic lithosphere. This sensitivity is studied in Fig. 8, where vertical displacement at the ice sheet margin $(\theta=\alpha)$ is shown for various values of $\alpha$ after three centuries since the onset of loading, keeping fixed the accretion rate of the ice sheet at $10 \mathrm{~cm} \mathrm{yr}^{-1}$. The results show two distinct behaviors with varying $\alpha$. For models REF, BRL and CAP, as well as for the elastic solution, displacements are weakly dependent upon the size of the load and their absolute value decreases with decreasing $\alpha$. On the contrary, when LVZ and BUR models are considered, it increases with decreasing $\alpha$ until $\alpha \sim 2^{\circ}$ (for this value, the horizontal extent of the load is comparable with the thickness of the LVZ), and suddenly decreases for smaller values of the load size, when the elastic lithosphere compensates the load by its flexural rigidity. For $\alpha \sim 2^{\circ}$, a significant divergence from the elastic values is observed. The largest effects are found for model LVZ18 and relatively small values of $\alpha$, when at the ice sheet margin the response of the asthenosphere enhances the elastic response by a factor of $\sim 10$. The rheological amplification effect decreases with increasing viscosity in the LVZ and is moderate for the BUR rheology.

\section{Conclusions}

Vertical and horizontal Love numbers exhibit different sensitivities to shallow upper mantle rheological layering. When the traditional
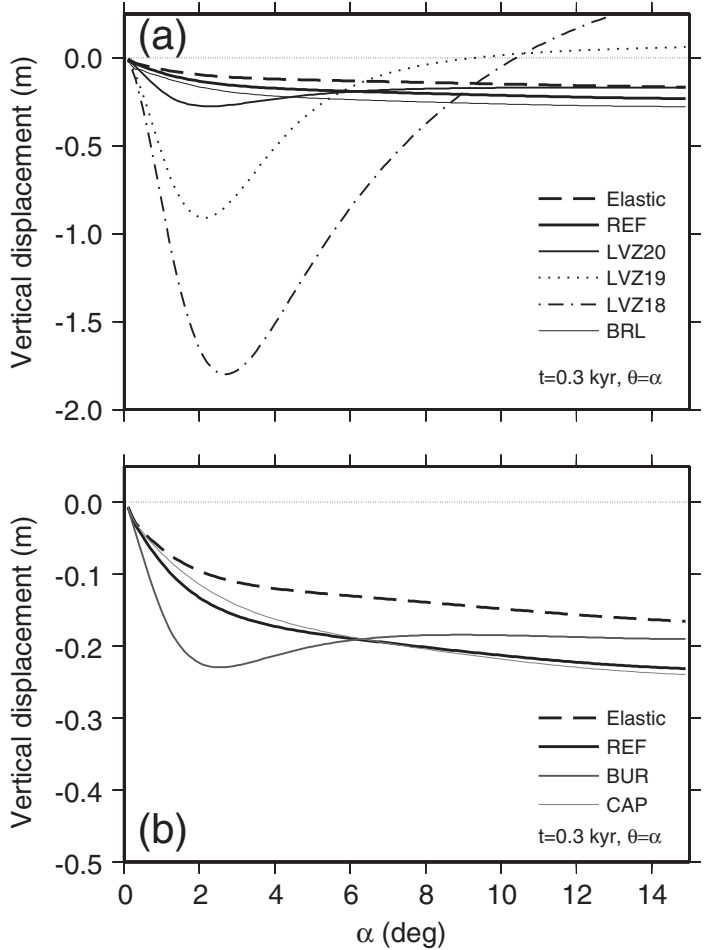

Fig. 8. Vertical displacement at the load margins $(\theta=\alpha)$ as a function of the loads halfamplitude $\alpha$. Displacements are computed at time $t=0.3 \mathrm{kyr}$. Note that the two frames have different vertical scales.

REF mantle viscosity profile is employed, we have found that the fundamental harmonics of degree two does not show any significant amplification relative to the elastic components on the decade to century time scale. Since these harmonics are directly related to variations of the inertia tensor (Lambeck, 1980), this indicates that short-term fluctuations of the Earth rotation forced by surface loading are substantially insensitive to shallow upper mantle rheology as far as its viscosity is close to the Haskell value of $10^{21} \mathrm{~Pa}$ s. On the century time scale, the RA factor does not vary substantially with harmonic degree in the range $2 \leq n \leq 128$, reaching a maximum amplitude of $\sim 1.5$.

Introducing a LVZ with Maxwell rheology and viscosity of $10^{18}$ or $10^{19} \mathrm{~Pa} s$ significantly alters the Love numbers at all wavelengths. At harmonic degree two, the response of the Earth departs from its elastic asymptote already at a decade scale. This is particularly evident for the horizontal Love number, which shows enhanced oscillations before reaching the fluid regime. In the presence of a LVZ, rheological amplification factors for vertical displacement at $t=1$ century peaks for harmonic degrees $30 \leq n \leq 40$ with values as large as RA 20. A more complex, bimodal response, is shown by the harmonics of horizontal displacement, which also change their sign with respect to the purely elastic solution. The two transient rheologies tested here, BUR and CAP, affect differently the Love numbers, with the largest amplifications obtained using BUR, but modest effects on the harmonic degree two Love numbers even on a century scale. Furthermore, on the time scales of interest here, a moderate rheological amplification (RA 1.5) of the long-wavelength elastic Love numbers is expected from the relaxation of crustal low viscosity layers, as we have verified using the model previously proposed by Barletta et al. (2006). The implementation of the PW formula has been useful to compute Love numbers for Earth models including thin, low viscosity crustal layers, even in the numerically challenging case of large ( $n \sim 100,000)$ harmonic degrees.

In the last part of the manuscript, we have modeled the response of the Earth to a simple parabolic cross-section surface load growing at a 
constant rate, with the purpose of evaluating deviations from the elastic response on time scales of a few centuries. In the vicinity of the load center, the elastic vertical displacements computed by the REF model always constitute a lower bound to values obtained using any other rheological model. In this region, after a few centuries since the beginning of loading, viscoelastic deformations exceed the elastic ones by a factor ranging between 1 and 4 . The largest effects are expected in the case of a very soft LVZ with viscosity $10^{18} \mathrm{~Pa}$ s. However, we have found that the sensitivity of vertical deformation to shallow upper mantle rheology is considerably more complex at the ice sheet margin, where vertical movements have a key-role in the assessment of present and future regional sea level variations (Sheperd and Wingham, 2007; IPCC, Climate Change, 2007). In these regions, a "softening" of the shallow upper mantle rheology does not necessarily imply a larger rate of deformation relative to the elastic case, as we have observed for LVZ20 models. The viscosity of the LVZ determines the pattern and the sign of vertical displacement at the ice margins, and may produce complex vertical movements before a steady state regime is attained after approximately one century since the onset of glacial instability. In the presence of a LVZ with viscosity $<10^{19} \mathrm{Pas}$, melting or accretion of relatively small $\left(\alpha \sim 2^{\circ}\right)$ ice sheets can produce vertical deformations exceeding the elastic ones by a factor of $\sim 10$ on time scales of a few centuries.

\section{Acknowledgments}

We are grateful to Francesco Mainardi for a number of illuminating discussions on various topics of linear viscoelasticity, to Valentina Barletta and to two anonymous reviewers for their very constructive comments. All the figures were drawn using the GMT public domain software (Wessel and Smith, 1998). To facilitate reproducibility of the results, the code ALMA is freely available from the author (email: giorgio.spada@gmail.com). Part of this work was supported by COST Action ES0701 "Improved constraints on models of Glacial Isostatic Adjustment". We acknowledge the ice2sea project, funded by the European Commission's 7th Framework Programme through grant number 226375 (ice2sea manuscript number 003).

\section{References}

Bamber, J.L., Riva, R.E.M., Vermeersen, L.L.A., LeBrocq, A.M., 2009. Reassessment of the potential sea-level rise from a collapse of the West Antarctic Ice Sheet. Science 324 doi:10.1126/science.1169335.

Barletta, V.R., Ferrari, C., Diolaiuti, G., Carnielli, T., Sabadini, R., Smiraglia, C., 2006. Glacier shrinkage and modeled uplift of the Alps. Geophysical Research Letters 33, L14307. doi:10.1029/2006GL026490.

Burgers, J.M., 1935. Mechanical considerations, model systems, phenomenologica theories of relaxation and of viscosity. In: Burgers, J.M. (Ed.), First Report on Viscosity and Plasticity, Nordemann Publishing Company, New York (1935).

Burov, E., Podladchkov, Y., Grandjean, G., Burg, J.P., 1999. Thermo-mechanical approach to validation of deep crustal and lithospheric structures inferred from multidisciplinary data: application to the Western and northern Alps. Terra Nova 11 (2-3), 124-131.

Caputo, M., 1967. Linear models of dissipation whose $Q$ is almost frequency independent. Geophysical Journal of the Royal Astronomical Society 13, 529-539.

Cathles, L.M., 1975. The Viscosity of the Earth's Mantle. Princeton University Press, N. J. 386 pp.

Christensen, R.M., 1982. Theory of Viscoelasticity. Academic Press, NY. 364 pp.

Di Donato, G., Mitrovica, J.X., Sabadini, R., Vermeersen, L.L.A., 2000. The influence of a ductile crustal zone on glacial isostatic adjustment; geodetic observables along the U.S. East Coast. Geophysical Research Letters 27, 3017-3020.

Dziewonski, A., Anderson, D.L., 1981. Preliminary reference Earth model. Physics of the Earth and Planetary Interiors 25, 297-356.

Farrell, W.E., 1972. Deformation of the earth by surface loads. Review of Geophysics and Space Physics 10, 761-797.

Fjeldskaar, W., 1994. Viscosity and thickness of the asthenosphere detected from the Fennoscandian uplift. Earth and Planetary Science Letters 126, 399-410.

Gaver, D.P., 1966. Observing stochastic processes and approximate transform inversion. Operative Research 14, 444-459.

Hanyk, L., 1999. Viscoelastic response of the Earth: initial-value approach. Ph. D Dissertation, Charles University, Prague, 137 pp.

Hanyk, L., Matyska, C., Yuen, D.A., 1999. Secular gravitational instability of a compressible viscoelastic sphere. Geophysical Research Letters 26, 557-560.
Haskell, N.A., 1935. The motion of a viscous fluid under a surface load. Physics 6 , 265-269.

IPCC, Climate Change, 2007. The Physical Science Basis. Cambridge University Press, Cambridge.

Ivins, E.R., Sammis, C.G., 1996. Transient creep of a composite lower crust. 1. Constitutive theory. Journal of Geophysical Research 101 (12), 27,981-28,004

James, T.S., Morgan, W.J., 1990. Horizontal motions due to post-glacial rebound. Geophysical Research Letters 17, 957-960.

Kendall, R., Mitrovica, J.X., Sabadini, R., 2003. Lithospheric thickness inferred from Australian post-glacial sea-level change: the influence of a ductile crustal zone. Geophysical Research Letters 30, 1461-1464.

Klemann, V., Wolf, D., 1999. Implications of a ductile crustal layer for the deformation caused by the Fennoscandian ice sheet. Geophysical Journal International 139, 216-226.

Koeller, R.C., 1984. Application of fractional calculus to the theory of viscoelasticity. Journal of Applied Mechanics 51, 299-307.

Körnig, M., Müller, G., 1989. Rheological models and interpretation of postglacial uplift. Geophysical Journal International 98, 243-253.

Lambeck, K., 1980. The Earth's Variable Rotation, Geophysical Causes and Consequences. Cambridge University Press, Cambridge. 449 pp.

Longman, M., 1962a. A Green's function for determining the deformation of the Earth under surface mass loads. 1. Theory. Journal of Geophysical Research 67, 845-850.

Longman, M., 1962b. A Green's function for determining the deformation of the Earth under surface mass loads. 2. Computations and numerical results. Journal of Geophysical Research 68, 485-496.

Love, A.E.H., 1909. The yielding of the Earth to disturbing forces. Proceedings of the Royal Society A 82, 73-88.

Mainardi, F., in press. Fractional Calculus and Waves in Linear Viscoelasticity. Imperial College Press, London, 340 pp.

Melini, D., Cannelli, V., Piersanti, A., Spada, G., 2008. Post-seismic rebound of a spherical Earth: new insights from the application of the Post-Widder inversion formula. Geophysical Journal International 174, 672-695. doi:10.1111/j.1365246X.2008.03847 x.

Mitrovica, J.X., Gomez, N., Clark, P.U., 2009. The sea-level fingerprint of West Antarctic collapse. Science 323, 753. doi:10.1126/science.1166510.

Munk, W.H., MacDonald, G.J.F., 1960. The Rotation of the Earth. Cambridge University Press, Cambridge. 323 pp.

Peltier, W.R., Drummond, R.A., Tushingham, A.M., 1986. Post-glacial rebound and transient lower mantle rheology. Geophysical Journal of the Royal Astronomical Society $87,79-116$.

Pollitz, F.F., 2003. Transient rheology of the uppermost mantle beneath the Mojave Desert, California. Earth and Planetary Science Letters 215, 89-104.

Post, E.L. 1930. Generalized differentiation. Transactions of the American Mathematical Society $32,723-781$.

Ranalli, G., 1995. Rheology of the Earth. Chapman and Hall, London. 413 pp.

Ranalli, G., Murphy, D., 1987. Rheological stratification of the lithosphere. Tectonophysics 132, 281-295.

Rümpker, G., Wolf, D., 1996. Viscoelastic relaxation of a Burgers half-space: implications for the interpretation of the Fennoscandia uplift. Geophysical Journal International 124, 541-555.

Sabadini, R., Yuen, D.A., Gasperini, P., 1988. Mantle rheology and satellite signatures from present-day glacial forcings. Journal of Geophysical Research 93 (B1), 437-447.

Schotman, H., 2008. Shallow-Earth Rheology from Glacial Isostasy and Satellite Gravity - a sensitivity analysis for GOCE. Thesis, Technische Universiteit Delft, 188 pp.

Schotman, H.H.A., Vermeersen, L.L.A., Wu, P., Drury, M.R., de Bresser, J.H.P., 2009. Constraints on shallow low-viscosity zones in Northern Europe from future GOCE gravity data. Geophysical Journal International 178, 65-84. doi:10.1111/j.1365246X.2009.04160.X

Schubert, G., Turcotte, D.L., Olson, P., 2001. Mantle Convection in the Earth and Planets. Cambridge University Press.

Sheperd, A., Wingham, D., 2007. Recent sea-level contributions of the Antarctic and Greenland ice sheets. Science 315, 1529-1532. doi:10.1126/science.1136776.

Smith, D.M., 1989. Efficient multiple-precision evaluation of elementary functions. Mathematics of Computation 52, 131-134.

Spada, G., 2003. The Theory Behind TABOO - A posT glAcial reBOund calculatOr. Samizdat Press, Golden, White River Junction, p. 109. http://samizdat.mines.edu/.

Spada, G., 2008. ALMA, a Fortran program for computing the visco-elastic Love numbers of a spherically symmetric planet. Computers and Geosciences 34 (6), 667-687. doi:10.1016/j.cageo.2007.12.001.

Spada, G., Boschi, L., 2006. Using the Post-Widder formula to compute the Earth's viscoelastic Love numbers. Geophysical Journal International 166, 309-321. doi:10.1111/j.1365-246X.2006.02995.x.

Spada, G., Stocchi, P., 2006. The Sea Level Equation, Theory and Numerical Examples. Aracne, Roma. ISBN: 88-548-0384-7, 96 pp.

Spada, G., Sabadini, R., Yuen, D.A., Ricard, Y., 1992. Effects on post-glacial rebound from the hard rheology in the transition zone. Geophysical Journal International 109 (2), 683-700. doi:10.1111/j.1365-246X.1992.tb00125.x.

Spada, G., Antonioli, A., Cianetti, S., Giunchi, C., 2006. Glacial isostatic adjustment and relative sea-level changes: the role of lithospheric and upper mantle heterogeneities in a 3-D spherical Earth. Geophysical Journal International 165 (2), 692-702. doi:10.1111/j.1365- 246X.2006.02969.x.

Stocchi, P., Spada, G., Cianetti, S., 2005. Isostatic rebound following the Alpine deglaciation: impact on the sealevel variations and vertical movements in the Mediterranean region. Geophysical Journal International 162. doi:10.1111/j.1365246X.2005.02653.X. 
Tushingham, A.M., Peltier, W.R., 1991. Ice-3G: a new global model of late Pleistocene deglaciation based upon predictions of postglacial sea-level change. Journal of Geophysical Research 96, 4497-4523.

Valko, P.P., Abate, J., 2004. Comparison of sequence accelerators for the Gaver method of numerical Laplace transform method. Computers and Mathematics with Applications 48, 629-636.

van der Wal, W., Schotman, H.H.A., Vermeersen, L.L.A., 2004. Geoid heights due to a crustal low viscosity zone in glacial isostatic adjustment modeling; a sensitivity analysis for GOCE. Geophysical Research Letters 31. doi:10.1029/2003GL019139.

Vermeersen, L.L.A., 2003. The potential of GOCE in constraining the structure of the crust and lithosphere from post-glacial rebound. Space Science Reviews 108, 105-113.

Vermeersen, L.L.A., Sabadini, R., 1997. A new class of stratified viscoelastic models by analytical techniques. Geophysical Journal International 129, 531-570.
Wessel, P., Smith, W.H.F., 1998. New, improved version of generic mapping tools released. Eos, Transactions, American Geophysical Union 79, 579.

Widder, D.V., 1934. The inversion of the Laplace integral and the related moment problem. Transactions of the American Mathematical Society Translations 36, 107-200.

Widder, D.V., 1946. The Laplace Transform. Princeton University Press, Princeton, NJ. 406 pp.

Wieczerkowski, K., Wolf, D., 1998. Viscoelastic Tidal Dissipation in Planetary Models. In: Ducarme, B., Paquet, P. (Eds.), Proceedings of the 13th International Symposium on Earth Tides. Observatoire Royal de Belgique, pp. 277-285.

Wu, P., Ni, Z., 1996. Some analytical solutions for the viscoelastic gravitational relaxation of a two-layer non-self-gravitating incompressible spherical earth. Geophysical Journal International 126, 413-436.

Yuen, D.A., Sabadini, R.C.A., Gasperini, P., Boschi, E., 1986. On transient rheology and glacial isostasy. Journal of Geophysical Research 91, 11,420-11,438. 